\title{
FUNCTIONAL EQUATIONS, TEMPERED DISTRIBUTIONS AND FOURIER TRANSFORMS
}

\author{
JOHN A. BAKER
}

\begin{abstract}
This paper introduces a method for solving functional equations based on the Fourier transform of tempered distributions.
\end{abstract}

\section{INTRODUCTION}

This paper concerns the study of complex valued functions, $f$, of several real variables satisfying a functional equation or system of functional equations of the following form:

$$
\sum_{k=0}^{m} a_{k} f\left(x+h_{k}\right)=q(x), \quad x \in \mathbf{R}^{n} .
$$

Here $a_{0}, \ldots, a_{m}$ are complex numbers, $h_{0}, \ldots, h_{m}$ belong to $\mathbf{R}^{n}$ and $q$ is a polynomial in $n$ variables with complex coefficients.

Our aim is to introduce a method for solving such systems based on Laurent Schwartz's theory of distributions and his theory of the Fourier transform of tempered distributions. We will show that, under certain assumptions on the $a$ 's and $h$ 's, if a function $f$ satisfies such a system and also satisfies a mild regularity condition (such as local integrability) then $f$ is almost everywhere equal to a polynomial.

In order to illustrate the method several examples are presented, many of which concern functional equations which have been studied extensively.

Consider, for example, the functional equation

$$
\sum_{k=0}^{m} f_{k}\left(x+y b_{k}\right)=q_{y}(x)
$$

where $b_{0}, \ldots, b_{m}$ are distinct real numbers, $f_{k}: \mathbf{R} \rightarrow \mathbf{C}$ for $0 \leq k \leq m$, $\varnothing \neq C \subseteq \mathbf{R}, q_{y}$ is a polynomial for each $y \in C$ and (\#) holds for all $x \in \mathbf{R}$ and $y \in C$. Kemperman [7] has considered this and related, more general, equations in considerable depth. For example, he showed that if (\#) holds with $f_{0}$ Lebesgue measurable and $C=\{j a+k b: 0 \leq j, k \in \mathbf{Z}, j+k \leq m\}$

Received by the editors September 10, 1987. 46F12.

1980 Mathematics Subject Classification (1985 Revision). Primary 39B40; Secondary 39A10,

This research was initiated while the author was still supported by NSERC Grant A7153. 
where $a, b$ are rationally independent real numbers then there exists a polynomial, $p$, such that $f_{0}(x)=p(x)$ for a.e. $x \in \mathbf{R}$. In case $f=f_{k}$ for $0 \leq k \leq m$, (\#) is a system of equations of type $(*)$. Many results of the present paper are similar to those obtained by Kemperman but our techniques are quite different. These techniques are also applied to find the tempered solutions of natural analogues of $(*)$ for distributions.

\section{NOTATION AND BACKGROUND FROM DISTRIBUTION THEORY}

The symbols $\mathbf{N}, \mathbf{Z}, \mathbf{R}$ and $\mathbf{C}$ denote the natural numbers, the integers, the real numbers and the complex numbers respectively. For $x=\left(x_{1}, \ldots, x_{n}\right)$ and $y=\left(y_{1}, \ldots, y_{n}\right)$ in $\mathbf{R}^{n}, x \cdot y=x_{1} y_{1}+\cdots+x_{n} y_{n}$ and $|x|=(x \cdot x)^{1 / 2}$. The closure of a subset $A$ of $\mathbf{R}^{n}$ will be denoted by $\bar{A}$.

While some of our notation is not standard, the results mentioned here concerning distributions are well known and can be found, for example, in Hörmander [6] or Rudin [11]. The space of all complex valued, $C^{\infty}$ functions on $\mathbf{R}^{n}$ will be denoted by $C_{n}^{\infty}$. We denote by $\mathscr{D}_{n}$ the space of test functions on $\mathbf{R}^{n}$ (members of $C_{n}^{\infty}$ with compact support). The space of Schwartz distributions on $\mathbf{R}^{n}$ will be denoted by $\mathscr{D}_{n}^{\prime}$. If $\phi \in \mathscr{D}_{n}$ then the support of $\phi$ will be denoted by $\operatorname{supp} \phi$ and if $u \in \mathscr{D}_{n}^{\prime}$ the support of $u$ will be denoted by supp $u$. If $f: \mathbf{R}^{n} \rightarrow \mathbf{C}$ is locally (Lebesgue) integrable on $\mathbf{R}^{n}$ and we let $\lambda_{f}(\phi)=\int_{\mathbf{R}^{n}} f(x) \phi(x) d x$ for $\phi \in \mathscr{D}_{n}$ then $\lambda_{f} \in \mathscr{D}_{n}^{\prime}$ : we refer to $\lambda_{f}$ is the regular distribution determined by $f$.

The space of all rapidly decreasing, complex valued functions on $\mathbf{R}^{n}$ (see Rudin [11, p. 168]) will be denoted by $\mathscr{S}_{n}$. The Schwartz space, or space of tempered distributions, on $\mathbf{R}^{n}$ will be denoted by $\mathscr{S}_{n}^{\prime}$. If $f: \mathbf{R}^{n} \rightarrow \mathbf{C}$ then we say that $f$ is temperate provided it is locally integrable and $\lambda_{f} \in \mathscr{S}_{n}^{\prime}$. For this it is sufficient that $f$ be measurable and have polynomial growth, that is, there exist $A>0$ and $m \in \mathbf{N}$ such that $|f(x)| \leq A\left(1+|x|^{m}\right)$ for all $x \in \mathbf{R}^{n}$. Every probability measure on $\mathbf{R}^{n}$ is a tempered distribution.

If $h \in \mathbf{R}^{n}$ and $f: \mathbf{R}^{n} \rightarrow \mathbf{C}$ we let $\tau_{h} f$ denote the function defined by $\left(\tau_{h} f\right)(x)=f(x+h)$ for all $x \in \mathbf{R}^{n}$; if $f$ is locally integrable so is $\tau_{h} f$. For any $h \in \mathbf{R}^{n}, \tau_{h}$ (restricted to $\mathscr{D}_{n}$ ) is a topological automorphism of $\mathscr{D}_{n}$ and a topological automorphism of $\mathscr{S}_{n}$. If $h \in \mathbf{R}^{n}, f: \mathbf{R}^{n} \rightarrow \mathbf{C}$ is locally integrable and $\phi \in \mathscr{D}_{n}$ then

$$
\left(\lambda_{\tau_{h} f}\right)(\phi)=\int_{\mathbf{R}^{n}} f(x+h) \phi(x) d x=\int_{\mathbf{R}^{n}} f(y) \phi(y-h) d y=\lambda_{f}\left(\tau_{-h} \phi\right) .
$$

This motivates the following definition. If $h \in \mathbf{R}^{n}$ and $u \in \mathscr{D}_{n}^{\prime}$ define $\tau_{h} u$ : $\mathscr{D}_{n} \rightarrow \mathbf{C}$ by

$$
\left(\tau_{h} u\right)(\phi)=u\left(\tau_{-h} \phi\right) \text { for } \phi \in \mathscr{D}_{n} .
$$

Notice that if $f: \mathbf{R}^{n} \rightarrow \mathbf{C}$ is locally integrable then $\tau_{h} \lambda_{f}=\lambda_{\tau_{h} f}$. It is easy to check that $\tau_{h} u \in \mathscr{D}_{n}^{\prime}$ whenever $h \in \mathbf{R}^{n}$ and $u \in \mathscr{D}_{n}^{\prime}$. In fact, for each $h \in \mathbf{R}^{n}$, 
$\tau_{h}$ is a topological automorphism of $\mathscr{D}_{n}^{\prime}$. Moreover, if $h \in \mathbf{R}^{n}$ then $\tau_{h} u \in \mathscr{S}_{n}^{\prime}$ for all $u \in \mathscr{S}_{n}^{\prime}$ and $\tau_{h}$ is topological automorphism of $\mathscr{S}_{n}^{\prime}$.

Observe that $(*)$ can be written

$$
\sum_{k=0}^{m} a_{k}\left(\tau_{h_{k}} f\right)(x)=q(x), \quad x \in \mathbf{R}^{n},
$$

and, if $f$ is locally integrable, $(*)$ holds and $u=\lambda_{f}$ then

$$
\sum_{k=0}^{m} a_{k}\left(\tau_{h_{k}} u\right)=\lambda_{q}
$$

If $\phi \in \mathscr{S}_{n}$ we will denote the Fourier transform of $\phi$ by $\hat{\phi}$. Thus

$$
\hat{\phi}(x)=\int_{\mathbf{R}^{n}} \phi(t) \exp (-i x \cdot t) d t \quad \text { for } x \in \mathbf{R}^{n} .
$$

It is a remarkable fact that the Fourier transform is a topological automorphism of $\mathscr{S}_{n}$. The Fourier transform of a tempered distribution, $u$, will be denoted by $\mathscr{F}$; it is defined dually by

$$
(\mathscr{F} u)(\phi)=u(\hat{\phi}) \text { for } \phi \in \mathscr{S}_{n} \text { and } u \in \mathscr{S}_{n}^{\prime} \text {. }
$$

If $h \in \mathbf{R}^{n}$ let $e_{h}(x)=\exp (i h \cdot x)$ for $x \in \mathbf{R}^{n}$.

Let $\mathscr{P}_{n}$ denote the set of all polynomial functions in $n$ real variables with complex coefficients.

The Dirac delta functional on $\mathbf{R}^{n}$ will be denoted by $\delta$. Thus $\delta(\phi)=\phi(0)$ for $\phi \in \mathscr{D}_{n}$.

\section{General Results}

Our main results will be deduced from the following lemmas, all of which are likely known, but whose short proofs are included for completeness.

Lemma 1 [11, p. 167]. If $u \in \mathscr{S}_{n}^{\prime}$ and $h \in \mathbf{R}^{n}$ then $\mathscr{F}\left(\tau_{h} u\right)=e_{h} \mathscr{F} u$.

Proof. Suppose $h \in \mathbf{R}^{n}, u \in \mathscr{S}_{n}^{\prime}$ and $\phi \in \mathscr{S}_{n}$. Then

$$
\left(\mathscr{F}\left(\tau_{h} u\right)\right)(\phi)=\left(\tau_{h} u\right)(\hat{\phi})=u\left(\tau_{-h}(\hat{\phi})\right) .
$$

But, clearly, $\widehat{e_{h} \phi}=\tau_{-h} \hat{\phi}$ so that

$$
\left(\mathscr{F}\left(\tau_{h} u\right)\right)(\phi)=u\left(\widehat{e_{h} \phi}\right)=(\mathscr{F} u)\left(e_{h} \phi\right)=\left(e_{h} \mathscr{F} u\right)(\phi)
$$

Lemma 2. If $u, v \in \mathscr{D}_{n}^{\prime}, F \in C_{n}^{\infty}$ and $F u=v$ then

$$
\operatorname{supp} u \subseteq\left\{x \in \mathbf{R}^{n}: F(x)=0\right\} \cup \operatorname{supp} v \text {. }
$$

Proof. Let $C=\left\{x \in \mathbf{R}^{n}: F(x)=0\right\} \cup \operatorname{supp} v$. Suppose $\phi \in \mathscr{D}_{n}$ and $C \cap \operatorname{supp} \phi=\varnothing$. It suffices to show that $u(\phi)=0$.

Since $C$ is closed, supp $\phi$ is compact and $C \cap \operatorname{supp} \phi=\varnothing$ there exist open subsets, $U$ and $V$, of $\mathbf{R}^{n}$ such that $\operatorname{supp} \phi \subseteq U, C \subseteq V, \bar{U} \cap \bar{V}=\varnothing$ and $\bar{U}$ 
is compact. Choose $\psi \in \mathscr{D}_{n}$ such that $\psi(x)=1$ for $x \in \bar{U}, \psi(x)=0$ for $x \in \bar{V}$ and let

$$
G(x)= \begin{cases}\psi(x) / F(x) & \text { if } x \in \mathbf{R}^{n} \text { and } F(x) \neq 0, \\ 0 & \text { if } x \in \mathbf{R}^{n} \text { and } F(x)=0 .\end{cases}
$$

Then $G \in \mathscr{D}_{n}$. Moreover $G(x) F(x)=\psi(x)=1$ for all $x \in \bar{U}$ so that $F G \phi=\phi$ since $\operatorname{supp} \phi \subseteq U$. Hence $u(\phi)=u(F G \phi)=(F u)(G \phi)=v(G \phi)$. But $\operatorname{supp}(G \phi) \subseteq \operatorname{supp} \phi$ and

$$
\operatorname{supp} v \cap \operatorname{supp} \phi \subseteq C \cap \operatorname{supp} \phi=\varnothing
$$

so that $\operatorname{supp} v \cap \operatorname{supp}(G \phi)=\varnothing$ and thus $v(G \phi)=0$. Hence $u(\phi)=0$.

Remark. An analogous result holds for distributions on any nonempty open subset of $\mathbf{R}^{n}$ and can be proved in the same way.

Lemma 3. If $u \in \mathscr{S}_{n}^{\prime}$ then $\operatorname{supp} \mathscr{F} u \subseteq\{0\}$ if and only if $u=\lambda_{p}$ for some $p \in \mathscr{P}_{n}$.

Proof. If $v \in \mathscr{D}_{n}^{\prime}$ then $\operatorname{supp} v \subseteq\{0\}$ if and only if $v$ is a finite linear combination of derivatives of the Dirac delta functional (see Rudin [11, pp. 149 and 150]). Any such distribution is tempered. But, if $p \in \mathscr{P}_{n}$ then $\mathscr{F}\left(\lambda_{p}\right)$ is a finite linear combination of derivatives of $\delta$ [11, p. 177]. The proof can be completed by noting that the Fourier transform is a bijection of $\mathscr{S}_{n}^{\prime}[11, \mathrm{p}$. 176].

Lemma 4. Suppose $a_{k} \in \mathbf{C}$ and $h_{k} \in \mathbf{R}^{n}$ for $0 \leq k \leq m, q \in \mathscr{P}_{n}, u \in \mathscr{S}_{n}^{\prime}$ and

$$
\sum_{k=0}^{m} a_{k} \tau_{h_{k}} u=\lambda_{q}
$$

Then supp $u \subseteq\{x: F(x)=0\} \cup\{0\}$ where

$$
F(x)=\sum_{k=0}^{m} a_{k} \exp \left(i h_{k} \cdot x\right) \quad \text { for } x \in \mathbf{R}^{n} .
$$

Proof. By Lemma 1, $\left(\sum_{k=0}^{m} a_{k} e_{h_{k}}\right) \mathscr{F} u=\mathscr{F}\left(\lambda_{q}\right)$. But, by Lemma 3,

$$
\operatorname{supp} \mathscr{F}\left(\lambda_{q}\right) \subseteq\{0\} \text {. }
$$

The result follows from Lemma 2.

Theorem 1. Suppose $\Gamma$ is a nonempty set. For each $\gamma \in \Gamma$ suppose $q_{\gamma} \in \mathscr{P}_{n}$, $m(\gamma)$ is a natural number, $a_{k}(\gamma) \in \mathbf{C}$ and $h_{k}(\gamma) \in \mathbf{R}^{n}$ for $0 \leq k \leq m(\gamma)$. For $\gamma \in \Gamma$ let $F_{\gamma}(x)=\sum_{k=0}^{m(\gamma)} a_{k} \exp \left(i h_{k}(\gamma) \cdot x\right)$ for $x \in \mathbf{R}^{n}$, let

$$
Z_{\gamma}=\left\{x \in \mathbf{R}^{n}: F_{\gamma}(x)=0\right\}
$$

and suppose $\bigcap_{\gamma \in \Gamma} Z_{\gamma} \subseteq\{0\}$.

(i) If $u \in \mathscr{S}_{n}^{\prime}$ and, for all $\gamma \in \Gamma$,

$$
\sum_{k=0}^{m(\gamma)} a_{k}(\gamma) \tau_{h_{k}(\gamma)} u=\lambda_{q}
$$


then $u=\lambda_{p}$ for some $p \in \mathscr{P}_{n}$ and

$$
\sum_{k=0}^{m(\gamma)} a_{k}(\gamma) p\left(x+h_{k}(\gamma)\right)=q_{\gamma}(x) \quad \text { for } \gamma \in \Gamma \text { and } x \in \mathbf{R}^{n} .
$$

(ii) If $f: \mathbf{R}^{n} \rightarrow \mathbf{C}, f$ is temperate and, for each $\gamma \in \Gamma$,

$$
\sum_{k=0}^{m(\gamma)} a_{k}(\gamma) f\left(x+h_{k}(\gamma)\right)=q_{\gamma}(x) \text { for a.e. } x \in \mathbf{R}^{n},
$$

then there exists $p \in \mathscr{P}_{n}$ such that $f(x)=p(x)$ for a.e. $x \in \mathbf{R}^{n}$ and such that (2) holds.

(iii) If, in addition to the hypotheses of (ii), $f$ is continuous then $f \in \mathscr{P}_{n}$. Proof. (i) Suppose $u \in \mathscr{S}_{n}^{\prime}$ and (1) holds. By Lemma 4,

$$
\operatorname{supp} \mathscr{F} u \subseteq \bigcap_{\gamma \in \Gamma}\left[Z_{\gamma} \cup\{0\}\right] \subseteq\{0\} \text {. }
$$

Thus, by Lemma $3, u=\lambda_{p}$ for some $p \in \mathscr{P}_{n}$.

(ii) Suppose $(1)^{\prime}$ holds and $f$ is temperate. If we let $u=\lambda_{f}$ then $u \in \mathscr{S}_{n}^{\prime}$ and (1) holds so that there exists $p \in \mathscr{P}_{n}$ such that $u=\lambda_{p}$. Thus $f(x)=p(x)$ for a.e. $x \in \mathbf{R}^{n}$ so that (2) holds for a.e. $x \in \mathbf{R}^{n}$ if $f$ is replaced by $p$. The continuity of $p$ implies that (2) holds for every $x \in \mathbf{R}^{n}$.

(iii) This follows easily from (ii).

\section{APPLications to functional eQuations}

Several of the examples to follow involve difference operators which we now define. If $A$ and $B$ are additive semigroups, $h \in A$ and $f: A \rightarrow B$ then $\triangle_{n} f: A \rightarrow B$ is defined by $\left(\Delta_{h} f\right)(x)=f(x+h)-f(x)$ for $x \in A$. Thus if $f: \mathbf{R}^{n} \rightarrow \mathbf{C}$ and $h \in \mathbf{R}^{n}$ then $\Delta_{h} f=\tau_{h} f-f$. The $m$ th iterate of the linear difference operator $\Delta_{h}$ is denoted by $\Delta_{h}^{m}$. We have

$$
\left(\Delta_{h}^{m} f\right)(x)=\sum_{k=0}^{m}(-1)^{m-k}\left(\begin{array}{c}
m \\
k
\end{array}\right) f(x+k h) \text { for } f: A \rightarrow B \text { and } x, h \in A .
$$

The functional equation

$$
\Delta_{h}^{m} f(x)=0
$$

has been studied extensively. It is known that if $A$ is an additive abelian semigroup, $Y$ is a rational vector space and $f: A \rightarrow Y$ then in order that (3) hold for all $x \in A$ and all $h \in A$ it is necessary and sufficient that there exists a constant $a_{0} \in Y$ and functions $a_{k}: A^{k} \rightarrow Y(1 \leq k \leq m-1)$ such that each $a_{k}$ is symmetric and additive (a homomorphism) in each variable and

$$
f(x)=a_{0}+\sum_{k=0}^{m-1} a_{k}^{*}(x) \text { for all } x \in A
$$


where $a_{k}^{*}(x)=a_{k}(x, x, \ldots, x)$ for $x \in A$ and $1 \leq k \leq m-1$ (see, for example, [4] or [10]). We aim to consider (3) for $f: \mathbf{R} \rightarrow \mathbf{C}$ but assuming it holds for only two (incommensurable) values of $h$. We will use the following special case of the result mentioned above which is also a well-known result from the calculus of finite differences (see e.g., [3, p. 477]).

Lemma 5. If $m \in \mathbf{N}$ and $g: \mathbf{Z} \rightarrow \mathbf{C}$ then

$$
\sum_{k=0}^{m}(-1)^{m-k}\left(\begin{array}{c}
m \\
k
\end{array}\right) g(n+k)=0 \text { for all } n \in \mathbf{Z}
$$

if and only if there exist $c_{0}, c_{1}, \ldots, c_{m-1} \in \mathbf{C}$ such that

$$
g(n)=\sum_{k=0}^{m-1} c_{k} n^{k} \text { for all } n \in \mathbf{Z} .
$$

Theorem 2. Let $0<a<b$ such that $a / b$ is irrational. Suppose $m, n \in \mathbf{N}$, $f: \mathbf{R} \rightarrow \mathbf{C}$ such that

$$
\triangle_{a}^{m} f(x)=0 \text { and } \triangle_{b}^{n} f(x)=0 \text { for every } x \in \mathbf{R}
$$

and $f$ is Lebesgue integrable on some interval of length ma. Then there exists $p \in \mathscr{P}_{1}$ with degree at most $m-1$ such that $f(x)=p(x)$ for a.e. $x \in \mathbf{R}$ and

$$
\triangle_{a}^{m} p(x)=0=\triangle_{b}^{n} p(x) \text { for all } x \in \mathbf{R} .
$$

Proof. Since every translation of $f$ satisfies the same sort of conditions as $f$, we may assume that $f$ is integrable on $[0, m a]$.

For $x \in[0, a)$ let $g_{x}(n)=f(x+n a)$ for $n \in \mathbf{Z}$. Then

$$
\sum_{k=0}^{m}(-1)^{m-k}\left(\begin{array}{c}
m \\
k
\end{array}\right) q_{x}(n+k)=\triangle_{a}^{m} f(x+n a)=0 \quad \text { for } x \in[0, a) \text { and } n \in \mathbf{Z} \text {. }
$$

Hence, by Lemma 5 , for each $x \in[0, a)$ there exist $c_{0}(x), \ldots, c_{m-1}(x) \in \mathbf{C}$ such that

$$
f(x+n a)=g_{x}(n)=\sum_{k=0}^{m-1} c_{k}(x) n^{k} \quad \text { for every } n \in \mathbf{Z} .
$$

By substituting $n=0, n=1, \ldots, n=m-1$ in (4) we obtain a system of $m$ linear equations which can be "solved" for $c_{0}(x), \ldots, c_{m-1}(x)$. Thus there exist $\gamma_{j k} \in \mathbf{C}$ for $0 \leq j, k \leq m-1$ such that

$$
c_{k}(x)=\sum_{j=0}^{m-1} \gamma_{j k} f(x+j a) \text { for } 0 \leq x<a \text { and } 0 \leq k \leq m-1 .
$$

In particular $c_{0}(x)=f(x)$ for $0 \leq x<a$. Since $f$ is integrable on $[0, m a]$, it follows that $c_{k}$ is integrable on $[0, a]$ for each $k=0,1, \ldots, m-1$. Thus, according to (4), $f$ is locally integrable on $\mathbf{R}$. 
If $n \in \mathbf{Z}$ and $n \neq 0$ then, according to (4),

$$
\begin{aligned}
\int_{n a}^{(n+1) a}|f(y)| d y & =\int_{0}^{a}|f(x+n a)| d x \leq \sum_{k=0}^{m-1}\left(\int_{0}^{a}\left|c_{k}(x)\right| d x\right)|n|^{k} \\
& \leq C \sum_{k=0}^{m-1}|n|^{k} \leq m C|n|^{m-1}
\end{aligned}
$$

where $\mathbf{C}=\max \left\{\int_{0}^{a}\left|c_{k}(x)\right| d x: 0 \leq k \leq m-1\right\}$.

Let $\varepsilon>0$. Suppose $\phi \in \mathscr{S}_{1}$ and $\left(1+|x|^{m+1}\right)|\phi(x)|<\varepsilon$ for all $x \in \mathbf{R}$. If $n \in \mathbf{N}$ then

$$
\begin{aligned}
\int_{n a}^{(n+1) a}|f(y) \phi(y)| d y & \leq m C n^{m-1} \max \{|\phi(y)|: n a \leq y \leq(n+1) a\} \\
& \leq m C n^{n-1} \varepsilon /\left(1+(n a)^{m+1}\right)
\end{aligned}
$$

so that for each $N \in \mathbf{N}$,

$$
\int_{1}^{(N+1) a}|f(y) \phi(y)| d y \leq m C \sum_{n=1}^{N} n^{m-1} /\left(1+(n a)^{m+1}\right)<C^{\prime} \varepsilon
$$

where $C^{\prime}=m C \sum_{n=1}^{\infty} n^{m-1} /\left(1+(n a)^{m+1}\right)$.

Similarly, there exists $C^{\prime \prime}>0$ such that

$$
\int_{-N a}^{0}|f(y) \phi(y)| d y \leq \varepsilon C^{\prime \prime} \quad \text { for every } N \in \mathbf{N} .
$$

But

$$
\int_{0}^{a}|f(y) \phi(y)| d y \leq C \max \{|\phi(y)|: 0 \leq y \leq a\}<\varepsilon C .
$$

Thus $\int_{\mathbf{R}}|f(y) \phi(y)| d y \leq\left(C+C^{\prime}+C^{\prime \prime}\right) \varepsilon$. It follows that if $\phi_{j} \rightarrow 0$ in $\mathscr{S}_{1}$ then $\lambda_{f}\left(\phi_{j}\right) \rightarrow 0$ in C. That is, $\lambda_{f} \in \mathscr{S}_{1}^{\prime}$. Let $u=\lambda_{f}$.

Now $0=\triangle_{a}^{m} u=\sum_{k=0}^{m-1}(-1)^{m-k}\left(\begin{array}{c}m \\ k\end{array}\right) \tau_{k a} u=0$ so that, by Lemma $1, F_{a} \mathscr{F} u=$ 0 where

$$
F_{a}(x)=\sum_{k=0}^{m-1}(-1)^{m-k}\left(\begin{array}{c}
m \\
k
\end{array}\right) \exp (\text { ikax })=(1-\exp (\text { iax }))^{m}
$$

for all $x \in \mathbf{R}$. Similarly, $F_{b} \mathscr{F} \hat{u}=0$ where $F_{b}(x)=(1-\exp (i b x))^{n}$ for all $x \in \mathbf{R}$. Now if $x \in \mathbf{R}$ and $F_{a}(x)=F_{b}(x)=0$ then $a x / 2 \pi$ and $b x / 2 \pi$ are integers and hence $x=0$ since $a / b$ is irrational. The existence of an appropriate $p$ follows from Theorem 1 . The assertion concerning the degree of $p$ follows either by substitution or by considering the restrictions of $p$ to $a \mathbf{Z}$ and $b \mathbf{Z}$ and using properties of difference operators which can be found in [3] or [4].

There is a vast literature concerning the quadratic equation

$$
f(x+y)+f(x-y)=2 f(x)+2 f(y) \text {. }
$$


It is known, [9], that if $f: \mathbf{R} \rightarrow C$ satisfies this equation for all $x, y \in \mathbf{R}$ and if $f$ is bounded on some subset of $\mathbf{R}$ having positive inner Lebesgue measure then there exists $c \in \mathbf{C}$ such that $f(x)=c x^{2}$ for all $x \in \mathbf{R}$. As a further illustration of our method we present

Theorem 3. Let $0<a<b$ such that $a / b$ is irrational. Suppose $\alpha, \beta \in \mathbf{C}$, $f: \mathbf{R} \rightarrow \mathbf{C}$

$$
\begin{aligned}
& f(x+a)+f(x-a)=2 f(x)+\alpha \quad \text { for all } x \in \mathbf{R}, \\
& f(x+b)+f(x-b)=2 f(x)+\beta \quad \text { for all } x \in \mathbf{R},
\end{aligned}
$$

and $f$ is integrable on an interval of length $3 a$. Then there exists $p \in \mathscr{P}_{1}$ such that $f(x)=p(x)$ for a.e. $x \in \mathbf{R}$ and the degree of $p$ is at most 2 .

Proof. On replacing $x$ by $x+a$ in the first of our two functional equations we find that $\triangle_{a}^{2} f(x)=f(x+2 a)-2 f(x+a)+f(x)=\alpha$ for all $x \in \mathbf{R}$ so that $\triangle_{a}^{3} f(x)=0$ for all $x \in \mathbf{R}$. Similarly $\triangle_{b}^{3} f(x)=0$ for all $x \in \mathbf{R}$. The result follows directly from the last theorem.

The next example involves a single equation.

Theorem 4. Suppose $\mu_{1}, \ldots, \mu_{m}>0, \sum_{k=1}^{m} \mu_{k}=1$. Suppose $h_{1}, \ldots, h_{m} \in \mathbf{R}^{n}$ such that if $x \in \mathbf{R}^{n}$ and $h_{k} \cdot x \in 2 \pi \mathbf{Z}$ for all $k=1, \ldots, m$ then $x=0$ (in case $n=1$ it suffices that $h_{i}, h_{j}$ be rationally independent for some $i$ and $j$ such that $1 \leq i<j \leq m)$. Let $q \in \mathscr{P}_{n}$.

(i) If $u \in \mathscr{S}_{n}^{\prime}$ and

$$
u=\sum_{k=0}^{m} \mu_{k} \tau_{h_{k}} u+\lambda_{q}
$$

then there exists $p \in \mathscr{P}_{n}$ such that $u=\lambda_{p}$ and

$$
p(x)=\sum_{k=1}^{m} \mu_{k} p\left(x+h_{k}\right)+q(x) \text { for all } x \in \mathbf{R}^{n} .
$$

(ii) If $f: \mathbf{R}^{n} \rightarrow \mathbf{C}, f$ is temperate and

$$
f(x)=\sum_{k=1}^{m} \mu_{k} f\left(x+h_{k}\right)+q(x) \text { for a.e. } x \in \mathbf{R}^{n}
$$

then there exists $p \in \mathscr{P}_{n}$ such that $f(x)=p(x)$ for a.e. $x \in \mathbf{R}^{n}$ and (6) holds.

Proof. Let $F(x)=1-\sum_{k=1}^{m} \mu_{k} \exp \left(i h_{k} \cdot x\right)$ for $x \in \mathbf{R}^{n}$. Suppose $x \in \mathbf{R}^{n}$ and $F(x)=0$. Then

$$
\left|\sum_{k=1}^{m} \mu_{k} \exp \left(i h_{k} \cdot x\right)\right|=1=\sum_{k=1}^{m} \mu_{k}=\sum_{k=1}^{m}\left|\mu_{k} \exp \left(i h_{k} \cdot x\right)\right| .
$$

Since $\mu_{k}>0$ for $1 \leq k \leq m$ we must have $\exp \left(i h_{k} \cdot x\right)=1$ for $1 \leq k \leq m$. Thus $h_{k} \cdot x \in 2 \pi \mathrm{Z}$ for $1 \leq k \leq m$ so that $x=0$. 
The result follows directly from Theorem 1 .

In [2] it was shown that if $f: \mathbf{R}^{2} \rightarrow \mathbf{R}$,

$$
f(x+h, y)+f(x-h, y)+f(x, y+h)+f(x, y-h)=4 f(x, y)
$$

for all $(x, y) \in \mathbf{R}^{2}$ and all $h \in \mathbf{R}$ and if $f$ is bounded on some set of positive planar Lebesgue measure then $f$ is a polynomial of degree at most 4. We aim to demonstrate a similar "mean value" result assuming the equation in question holds for only four values of $h(a, b, 2 a$ and $2 b$ with $a, b$ rationally independent). Notice that the equation has an interesting geometric interpretation. As noted in [2], (8) can be viewed as a difference analogue of the Laplace equation. by

For $h \in \mathbf{R}$ and $f: \mathbf{R}^{2} \rightarrow \mathbf{C}$ define the "partial differences", $\Delta_{h^{1}} f$ and $\Delta_{h^{2}} f$

$$
\begin{aligned}
& \left(\triangle_{h^{1}} f\right)(x, y)=f(x+h, y)-f(x, y) \text { and } \\
& \left(\triangle_{h^{2}} f\right)(x, y)=f(x, y+h)-f(x, y) \text { for all }(x, y) \in \mathbf{R}^{2} .
\end{aligned}
$$

The following lemma was inspired by geometric considerations from [2].

Lemma 6. Suppose $G$ and $H$ are additive abelian groups. For $f: G^{2} \rightarrow H$ and $h \in G$ define $S_{h} f: G^{2} \rightarrow H$ and $T_{h} f: G^{2} \rightarrow H$ by

$$
\begin{aligned}
\left(S_{h} f\right)(x, y)= & f(x+h, y)+f(x-h, y)+f(x, y+h)+f(x, y-h)-4 f(x, y), \\
\left(T_{h} f\right)(x, y)= & f(x+h, y+h)+f(x-h, y+h)+f(x+h, y-h) \\
& +f(x-h, y-h)-4 f(x, y)
\end{aligned}
$$

for all $x, y \in G$. Then for all $h \in G$ and all $f: G^{2} \rightarrow H$,

$$
\begin{aligned}
& 2 T_{h} f=S_{h}\left(S_{h} f\right)+8 S_{h} f-S_{2 h} f, \\
& 2 S_{h} f=T_{h}\left(T_{h} f\right)+8 T_{h} f-T_{2 h} f,
\end{aligned}
$$

(iii) $f(x+2 h, y)-4 f(x+h, y)+6 f(x, y)-4 f(x-h, y)+f(x-2 h, y)$

$$
=\left(S_{h} f\right)(x+h, y)+\left(S_{h} f\right)(x-h, y)-\left(T_{h} f\right)(x, y) \text { for all } x, y \in G
$$

(iv) $f(x, y+2 h)-4 f(x, y+h)+6 f(x, y)-4 f(x, y-h)+f(x, y-2 h)$

$$
=\left(S_{h} f\right)(x, y+h)+\left(S_{h} f\right)(x, y-h)-\left(T_{h} f\right)(x, y) \text { for all } x, y \in G,
$$

(v) $\triangle_{h^{1}}^{4} f(x, y)=\left(S_{h} f\right)(x+3 h, y)+\left(S_{h} f\right)(x+h, y)-\left(T_{h} f\right)(x+2 h, y)$ for all $x, y \in G$

and

(vi) $\Delta_{h^{2}}^{4} f(x, y)=\left(S_{h} f\right)(x, y+3 h)+\left(S_{h} f\right)(x, y+h)-\left(T_{h} f\right)(x, y+2 h)$ for all $x, y \in G$. 
Proof. The proofs of (i)-(iv) involve only tedious calculations. By replacing $x$ by $x+2 h$ in (iii) we obtain (v). On replacing $y$ by $y+2 h$ in (iv) we obtain (vi).

Theorem 5. Suppose $f: \mathbf{R}^{2} \rightarrow \mathbf{C}, 0<a<b, a / b$ is irrational and $S_{a} f=$ $S_{2 a} f=S_{b} f=S_{2 b} f=0$. If $f$ is integrable on $\left[x_{0}, x_{0}+4 a\right] \times\left[y_{0}, y_{0}+4 a\right]$ for some $\left(x_{0}, y_{0}\right) \in \mathbf{R}^{2}$ then $f$ is a polynomial of degree at most 4 .

Proof. Since $S_{a} f=S_{2 a} f$, from (i) of Lemma 6 we find that $T_{a} f=0$. Then, since $S_{a}=0$, (ii) of Lemma 6 implies that $T_{2 a} f=0$ as well. Thus, by (v) and (vi) of Lemma 6 ,

$$
\triangle_{a^{1}}^{4} f(x, y)=0 \text { and } \triangle_{a^{2}}^{4} f(x, y)=0 \text { for all }(x, y) \in \mathbf{R}^{2} .
$$

Similarly, since $S_{b} f=S_{2 b} f=0$,

$$
\triangle_{b^{1}}^{4} f(x, y)=0 \text { and } \triangle_{b^{2}}^{4}(x, y)=0 \text { for all } x, y \in \mathbf{R}^{2} .
$$

Choose measurable subsets $A$ and $B$ of $\left[x_{0}, x_{0}+4 a\right]$ and $\left[y_{0}, y_{0}+4 a\right]$ respectively such that $A$ and $B$ have Lebesgue measure $4 a, x \rightarrow f(x, y)$ is integrable on $\left[x_{0}, x_{0}+4 a\right]$ for every $y \in B$ and $y \rightarrow f(x, y)$ is integrable on $\left[y_{0}, y_{0}+4 a\right]$ for every $x \in A$.

According to Theorem 2, for each $y \in B$ there exist $c_{j}(y) \in \mathbf{C}, 0 \leq j \leq 3$ such that

$$
f(x, y)=c_{0}(y)+c_{1}(y) x+\cdots+c_{3}(y) x^{3} \text { for a.e } x \in \mathbf{R} .
$$

Similarly, for each $x \in A$ there exist $d_{k}(x), 0 \leq k \leq 3$, such that

$$
f(x, y)=d_{0}(x)+d_{0}(x) y+\cdots+d_{3}(x) y^{3} \text { for a.e. } y \in \mathbf{R} .
$$

Thus there exist measurable subsets $C$ and $D$ of $A$ and $B$ respectively each having Lebesgue measure $4 a$ and such that

$$
f(x, y)=\sum_{j=0}^{3} c_{j}(y) x^{j}=\sum_{k=0}^{3} d_{k}(x) y^{k} \text { for all }(x, y) \in C \times D .
$$

It follows that there exist $a_{j k} \in \mathbf{C}(0 \leq j, k \leq 3)$ such that

$$
f(x, y)=\sum_{j, k=0}^{3} a_{j k} x^{j} y^{k}
$$

for $x \in C$ and $y \in D$.

But for each $y \in D, \triangle_{a^{1}}^{4} f(x, y)=0$ for all $x \in \mathbf{R}$. It follows that (9) holds for all $x \in \mathbf{R}$ and $y \in D$. But $\triangle_{a^{2}}^{4} f(x, y)=0$ for all $x, y \in \mathbf{R}$ and thus it follows that (9) holds for all $x, y \in \mathbf{R}$.

We have shown that $f$ is a polynomial of degree at most 6 . The fact that it has degree at most 4 follows by substitution. In fact, it can be shown that $f$ is a harmonic polynomial. 
As a last illustration of the technique we will show that the termperate solutions of a generalization of (8) to $\mathbf{R}^{n}$ are polynomials. In what follows $\left\{\beta_{1}, \ldots, \beta_{n}\right\}$ is the usual basis for $\mathbf{R}^{n}$. We consider the equation

$$
\sum_{k=1}^{n} f\left(x+h \beta_{k}\right)+f\left(x-h \beta_{k}\right)=2 n f(x) .
$$

Notice that (10) has an interesting geometric interpretation where $n=1,2$ or 3 and it can be thought of as a difference analogue of the Laplace equation in $n$ dimension.

Theorem 6. Suppose $0<a<b, a / b$ is irrational, $f: \mathbf{R}^{n} \rightarrow \mathbf{C}$ and (10) holds for a.e. $x \in \mathbf{R}^{n}$ if $h=a$ or $h=b$. If $f$ is temperate then there exists $p \in \mathscr{P}_{n}$ such that $f(x)=p(x)$ for a.e. $x \in \mathbf{R}^{n}$.

Proof. Suppose $f$ is temperate and let

$$
F_{a}(x)=\sum_{k=1}^{n}\left\{\exp \left(i a \beta_{k}\right)+\exp \left(-i a \beta_{k}\right)\right\}-2 n
$$

and

$$
F_{b}(X)=\sum_{k=1}^{n}\left\{\exp \left(i b \beta_{k}\right)+\exp \left(-i b \beta_{k}\right)\right\}-2 n \text { for } x \in \mathbf{R}^{n} .
$$

If $x \in \mathbf{R}^{n}$ and $F_{a}(x)=F_{b}(x)=0$ then, by an argument like that used in the proof of Theorem $4, x=0$. The assertion follows from Theorem 1 .

\section{REMARKS}

Other distributional techniques have been used in the study of functional equations. See, for example, [2, 5 and 12]. Fourier analysis on groups has recently been used by Székelyhidi [13] in order to find the almost periodic solutions to certain functional equations which are closely related to the kind of equation considered here.

It is not difficult to obtain variants of Theorems 2 and 3 for functions of several real variables. Theorem 6 can be reformulated to obtain, in a similar way, an analogous result for tempered distributions.

As we have illustrated, it is often enlightening to consider a functional equation in the sense of Aczél [1] as a system of functional equations in a single variable in the sense of Kuczma [8].

\section{REFERENCES}

1. J. Aczél, Lectures on functional equations and their applications, Academic Press, New York and London, 1966.

2. J. Aczél, H. Haruki, M. A. McKiernan and G. N. Sakovič, General and regular solutions of functional equations characterizing harmonic polynomials, Aequationes Math. 7 (1968), 37-53.

3. D. L. Bentley and K. L. Cooke, Linear algebra with differential equations, Holt, Rinehart and Winston, New York, 1973. 
4. D. Ž. Djoković, $A$ representation theorem for $\left(X_{1}-1\right)\left(X_{2}-1\right) \cdots\left(X_{n}-1\right)$ and its applications, Ann. Polon. Math. 22 (1969), 189-198.

5. I. Fenyö, Über eine Lösungsmethode gewisser Funktionalgleichungen, Acta Math. Acad. Sci. Hungar. 7 (1956), 383-396.

6. Lars Hörmander, The analysis of linear partial differential operators. I, Springer-Verlag, Berlin, Heidelberg, New York and Tokyo, 1983.

7. J. H. B. Kemperman, A general functional equation, Trans. Amer. Math. Soc. 86 (1957), 28-56.

8. Marek Kuczma, Functional equations in a single variable, Monogr. Math. 46, PWN, Warsaw, 1968.

9. S. Kurepa, On the quadratic functional, Acad. Serbe Sci. Publ. Inst. Math. 13 (1959), 58-72.

10. S. Mazur and W. Orlicz, Grundlegende Eigenschaften der polynomischen Operation, Studia Math. 5 (1934), 50-68 and 179-189.

11. Walter Rudin, Functional analysis, McGraw-Hill, New York, 1973.

12. H. Swiatak, On the regularity of the distributional and continuous solutions of the functional equations $\sum_{i=1}^{k} a_{i}(x, t) f\left(x+\phi_{i}(t)\right)=b(x, t)$, Aequationes Math. 1 (1968), 6-19.

13. L. Székelyhidi, Almost periodicity and functional equations, Aequationes Math. 26 (1983), 163-175.

Department of Pure Mathematics, University of Waterloo, Waterloo, Ontario, CANADA N2L 3G1 